\title{
A Review on Plant Sources for Nano Biopesticide Production
}

\author{
Saranya Krishnamurthy ${ }^{1(\mathbb{D})}$, Manivasagan Veerasamy ${ }^{1}\left(\mathbb{D}\right.$, Gopi Karruppaya ${ }^{1, *}(\mathbb{D})$ \\ ${ }^{1}$ Department of Biotechnology, Adhiyamaan College of Engineering, Hosur, Tamil Nadu, India-635109 \\ *Correspondence: gopikarruppaya17@gmail.com;
}

Received: 16.06.2020; Revised: 4.07.2020; Accepted: 5.07.2020; Published: 7.07.2020

\begin{abstract}
Biopesticides are substances or mixtures of biological substances used to prevent, destroy, kill, control, or mitigate pests. Pesticide plants are also known as botanical pesticides, are derived naturally from plants. The use of chemical pesticides led to conflicts like environmental degradation and health issues like cancer, adverse effects on immune systems, neurological disorder, and metabolic diseases such as diabetes, endocrine system disruption, and infertility. Biopesticides are a type of pesticides obtained from natural sources such as animals, plants, bacteria, and certain minerals. Nanotechnology is a developing field dealing with materials having a size of $10^{-9}$. Farmers have been using this technology for proper plant growth stimulation, diagnosis of plant disease, and pest control. This paper briefly explains some evidence of plant species used to control pests and the presence of nanoparticles in that species for pest control are reviewed from different articles. These observations, perceptions, and notions provide an idea for future research to help the environment and humankind.
\end{abstract}

Keywords: Biopesticide; Nanoparticles; Pest control; Medicinal plants; Nanotechnology.

(C) 2020 by the authors. This article is an open-access article distributed under the terms and conditions of the Creative Commons Attribution (CC BY) license (https://creativecommons.org/licenses/by/4.0/).

\section{Introduction}

Biopesticides are substances or mixtures of biological substances used to prevent, destroy, kill, control, or mitigate pests. Pesticide plants are also known as botanical pesticides, are derived naturally from plants. Plants having pesticide activity are an ancient form of controlling pests. They act as a natural defense against pests developed over millions of years. Many plant species produce chemicals that affect pests. Plants having pesticide activity have been used for thousands of years. They were used widely in commercial agriculture up to the 1940s until chemical pesticides were developed. The use of chemical pesticides led to conflicts like environmental degradation and health issues like cancer, adverse effects on immune systems, neurological disorder, and metabolic diseases such as diabetes, endocrine system disruption, and infertility. Since biopesticides are now a small part of the overall pesticide market due to the development of chemical pesticides, the present environmental movement has provided a platform for the emergence of biopesticides. Nanotechnology is a developing field dealing with materials having a size of $10^{-9}$. Farmers have been using this technology for proper plant growth stimulation, diagnosis of plant disease, and pest control [1]. Chemical pesticides have various effects. It is estimated that due to insecticide poisoning, 200 deaths are occurring every year in the USA. WHO estimated that every year there are 25 million cases of pesticide poisoning, and 20,000 deaths are occurring in developing countries [2]. Because of the high exposure of chemical pesticides, the Netherlands rejected 130 containers of fresh 
grapes sent from India [3]. A global ban was called by the World Wildlife Fund on the production and use of DDT by 2007 [4].

\section{Nanoparticles based biopesticides}

Biopesticides are one of the types of pesticides obtained from natural resources such as animals, plants, bacteria, and certain minerals. They include fungi such as Beauveria sp., bacteria such as Bacillus sp., and neem extract. Likewise, canola oil and baking soda have applications of controlling pests, so they are known as biopesticides. From the above sources, both plants and microbes are employed in the synthesis of nanoparticles. For example, nanoparticles such as silver, nickel, cobalt, zinc, and copper are synthesized from plants, and they are employed in controlling pests [5]. Biopesticides are less harmful and eco-friendly in nature. They are employed to target only specific pests and decompose at a faster rate. It indicates the lower exposure and helps in controlling pollution. They are cost-effective and are biodegradable. By considering these advantages, a biopesticide is used for nanoparticle synthesis and plays an important role in agriculture for the welfare of human beings and also to our environment.

Nanotechnology involves nanoparticles (NPs) having a size of $100 \mathrm{~nm}$ or less or 10 and 1,000 nm. Metallic Nanoparticles are mostly synthesized from metals such as gold, silver, copper, and platinum. Nanotechnology is an emerging field in modern research. They are developing day by day, including life sciences, especially biomedical devices and biotechnology [6]. Nanotechnology has many applications in the field of chemical, agricultural, medical, and biological and cosmetics industries. In the beginning, mesoporous silica nanoparticles (MSNs) were used as a water-soluble pesticide against validamycin [7]. Alumina nanoparticles were used against two major insect pests in stored food, such as Sitophilus oryzae L. and Rhyzopertha dominica. Silver nanoparticles are used in packing food, clothing, disinfectant, and several household appliances. They also play an important role in treating diseases and prevent various health issues. The recent chemical researches show that the plant kingdom is a rich source of organic compounds. The nanoparticle-based biopesticide production process was mentioned in figure.1. Most of the plants are used as medicine, pesticides, and some are known as hallucinogens. Still, there is a lack of knowledge in this research. Hence, we should focus on plant species with uses reported in ethnobotanical and anthropological literature. This paper briefly explains some evidence of plant species used to control pests is reviewed from different articles. Their observations, perceptions, and notions provide an idea for future research to help the environment and humankind.

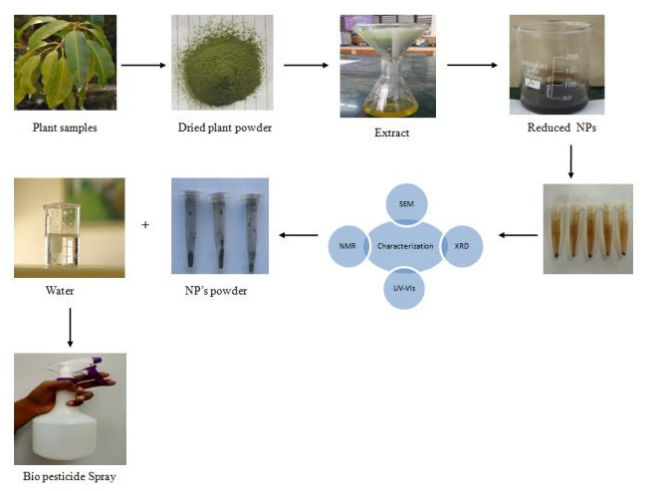

Figure 1. Nanoparticle-based biopesticide production process. 


\section{Plant-derived nanoparticle-based biopesticide}

Plants are one of the important sources of nanoparticles based biopesticides, and it contains different types of nanoparticles as a pesticide component in agriculture applications. Table 1 listed the biopesticide plants with scientific names, common names, types of nanoparticles present, insect or pest against which the plant is used, and plant parts used is listed.

Table 1.Plant-derived nanoparticle-based biopesticides.

\begin{tabular}{|c|c|c|c|c|}
\hline Plant name & Common name & NPs Present & Part of the plant used & Application \\
\hline Acorus calamus & Sweet flag & $\mathrm{Au}, \mathrm{Ag}$ & Root & Insecticide [8] \\
\hline Agave Americana & Sentry plant & $\mathrm{Ag}$ & Leaf & White ants [9] \\
\hline Ageratum conyzoides & Chickweed & $\mathrm{Ag}$ & Leaf & Lice in hair [10] \\
\hline Albizia lebbeck & Lebbeck & $\mathrm{Ag}, \mathrm{Ni}, \mathrm{Fe}$ & Seed,leaf,bark and root & Insecticide [11] \\
\hline Albizia procera & White siris & $\mathrm{Zn}, \mathrm{Ag}, \mathrm{Cu}$ & Leaf & Insecticide [12] \\
\hline Aloe secundiflora & $\begin{array}{l}\text { Newcastle in } \\
\text { chicken }\end{array}$ & $\mathrm{Cd}, \mathrm{Ag}$ & Sap & Insecticide[13] \\
\hline $\begin{array}{l}\text { Alysicarpus } \\
\text { bupleurifolius }\end{array}$ & Alyce clover & $\mathrm{Ag}, \mathrm{P}$ & Whole plant & $\begin{array}{l}\text { Bed bugs, white } \\
\text { ants [14] }\end{array}$ \\
\hline Anacardium occidentale & Kaaju & $\begin{array}{c}\mathrm{Au}, \mathrm{Ag}, \mathrm{Cu} \\
\mathrm{Pt}\end{array}$ & Shell oil & $\begin{array}{c}\text { White ants, } \\
\text { insecticide [15] }\end{array}$ \\
\hline Anamirta cocculus & Indian berry & $\mathrm{Zn}, \mathrm{Ag}, \mathrm{Au}$ & Fruit & Insecticide [16] \\
\hline Annona reticulate & Wild-sweetsop & $\mathrm{Ag}$ & Leaf, seed, and bark & $\begin{array}{c}\text { Body lice, } \\
\text { insecticide [17] }\end{array}$ \\
\hline Annona squamosa & Sugar apple & $\mathrm{Ag}, \mathrm{Fe}$ & $\begin{array}{l}\text { Seed, stem, bark, leaf, } \\
\text { and fruit }\end{array}$ & $\begin{array}{c}\text { Body lice, } \\
\text { insecticide [18] }\end{array}$ \\
\hline Arisaema tortuosum & Whipcord cobra lily & $\mathrm{Ag}, \mathrm{Au}, \mathrm{Zn}$ & Tubers & Insecticide [19] \\
\hline Aristolochia bracteolate & Worm killer & $\mathrm{Ag}$ & Juice & Insecticide [20] \\
\hline Artemisia japonica & Mugwort & $\mathrm{Ag}, \mathrm{Au}, \mathrm{Fe}$ & Whole plant & $\begin{array}{c}\text { Insecticide, } \\
\text { housefly repellant } \\
{[21]}\end{array}$ \\
\hline Artemisia nilagirica & Wormwood & $\mathrm{Ag}$ & Leaf & $\begin{array}{c}\text { Insect repellant, } \\
\text { prevent moths [22] }\end{array}$ \\
\hline Azadirachta indica & Neem tree & $\mathrm{Ag}, \mathrm{Cu}$ & Whole plant & $\begin{array}{l}\text { Insecticide, rice and } \\
\text { wheat weevil [23] }\end{array}$ \\
\hline Bambusa arundinacea & Bambusabambos & $\mathrm{Ag}$ & Shoot & $\begin{array}{c}\text { Kill mosquito larvae } \\
{[24]}\end{array}$ \\
\hline Bidens pilosa & Black jack & $\mathrm{Ag}, \mathrm{Au}$ & Leaves & Aphids [25] \\
\hline Blumea eriantha & Buradi & $\mathrm{Zn}$ & Whole plant & $\begin{array}{c}\text { Mosquito } \\
\text { repellant[26] }\end{array}$ \\
\hline Boswellia serrata & Indian frankincense & $\mathrm{Ag}$ & Gum & $\begin{array}{c}\text { Fumigation repel } \\
\text { houseflies and } \\
\text { mosquitoes [27] }\end{array}$ \\
\hline Brassica campestris & Field mustard & $\mathrm{Ag}, \mathrm{Zn}$ & Seed oil & Beetles [28] \\
\hline Butea monosperma & Sacred tree & $\mathrm{Ag}, \mathrm{Au}, \mathrm{Zn}$ & Seed and flower extract & White ants [29] \\
\hline Calotropis procera & Rubber bush & $\mathrm{Ag}, \mathrm{Zn}, \mathrm{Ni}, \mathrm{Fe}$ & Leaf & $\begin{array}{c}\text { Larvicidal, } \\
\text { insecticide [30] } \\
\end{array}$ \\
\hline Cannabis sativa & Kumbhi & $\mathrm{Au}, \mathrm{Ag}$ & Whole plant and leaf & Bugs \& pests [31] \\
\hline Capsicum annuum & Hot pepper & $\mathrm{Cu}, \mathrm{Ag}, \mathrm{Au}$ & Fruit , leaf & $\begin{array}{l}\text { Thrips, aphids and } \\
\text { white flies [32] }\end{array}$ \\
\hline Careya arborea & Karanda & $\mathrm{Ag}$ & Root, bark and leaf & Kill leeches [33] \\
\hline Carica papaya & Pawpaw & $\mathrm{Ag}, \mathrm{Zn}$ & Leaves, seeds & Several [34] \\
\hline Carissa congesta & Hemp & $\mathrm{Ag}$ & Root and bark & $\begin{array}{l}\text { Vet worms in } \\
\text { wounds [35] }\end{array}$ \\
\hline Cassia hirsute & Cassia & $\mathrm{Ag}, \mathrm{Zn}$ & Bark & Insecticide [36] \\
\hline Cassytha filiformis & Love vine & $\mathrm{Ag}, \mathrm{Cu}, \mathrm{Mg}$ & Whole plant & Insecticide [37] \\
\hline Catunaregam spinosa & $\begin{array}{c}\text { Mountain } \\
\text { pomegranate }\end{array}$ & $\mathrm{Sn}, \mathrm{Zn}, \mathrm{Ni}$ & Fruit & Insecticide [38] \\
\hline Cinnamomum camphora & Camphor tree & $\mathrm{Ag}, \mathrm{Au}, \mathrm{Pt}, \mathrm{Pd}$ & Bark powder & $\begin{array}{c}\text { Protect clothes } \\
\text { against insects [39] }\end{array}$ \\
\hline Citrus limon & Lemon & $\mathrm{Ag}, \mathrm{Au}$ & Dried leaf & $\begin{array}{l}\text { Wheat weevil and } \\
\text { flour beetle [40] }\end{array}$ \\
\hline Commiphora wightii & Indian bdellium tree & $\mathrm{Ag}$ & Resin & $\begin{array}{c}\text { Mosquito repellant } \\
{[41]}\end{array}$ \\
\hline
\end{tabular}




\begin{tabular}{|c|c|c|c|c|}
\hline Plant name & Common name & NPs Present & Part of the plant used & Application \\
\hline Cordia latifolia & Sebestan plum & $\mathrm{Ag}$ & Leaves & $\begin{array}{l}\text { Maize weevil, } \\
\text { butterfly [42] }\end{array}$ \\
\hline Corypha umbraculifera & Talipot palm & $\mathrm{Cu}, \mathrm{Ag}$ & Young fruit & Insect repellant [43] \\
\hline Croton roxburghii & Croton & $\mathrm{Ag}$ & Seed & Insecticide [44] \\
\hline Cucumis melo & Muskmelon & $\mathrm{Ag}$ & Leaf & Kill lice [45] \\
\hline Cucumis sativus & Cucumber & $\mathrm{Ag}, \mathrm{Cu}, \mathrm{Zn}$ & Rhizome & $\begin{array}{c}\text { Kill lice and insects } \\
{[46]}\end{array}$ \\
\hline Curcuma longa & Turmeric & $\mathrm{Ag}, \mathrm{Zn}$ & Rhizome & $\begin{array}{c}\text { Drive away ants } \\
{[47]}\end{array}$ \\
\hline Cuscuta reflexa & Amar bel & $\mathrm{Cu}, \mathrm{Ag}$ & Whole plant & Kill lice [48] \\
\hline Cymbopogan nardus & Citronella grass & $\mathrm{Ag}$ & whole plant & $\begin{array}{c}\text { Mosquito repellant } \\
{[49]}\end{array}$ \\
\hline Derris scandens & Gewel vine & $\mathrm{Ag}$ & leaf, bark & Insecticide [50] \\
\hline Derris trifoliate & Karanjvel & $\mathrm{Ag}$ & Bark & Insecticide [51] \\
\hline Desmodium triflorum & Tick clover & $\mathrm{Ag}, \mathrm{Cu}, \mathrm{Au}$ & Whole plant & Insecticide [52] \\
\hline Dioscorea hispida & Asiatic bitter yam & $\mathrm{Ag}$ & Bark & Insecticide [53] \\
\hline Duranta erecta & Brazilian skyflower & $\mathrm{Ag}, \mathrm{Zn}$ & Whole plant & Insecticide [54] \\
\hline Euphorbia antiquorum & Spurge & $\mathrm{Ag}$ & Milky juice & $\begin{array}{l}\text { Maggots in wound } \\
{[55]}\end{array}$ \\
\hline $\begin{array}{l}\text { Euphorbia } \\
\text { dracunculoides }\end{array}$ & Dragon spurge & $\mathrm{Ag}$ & Latex & Kills lice [56] \\
\hline Euphorbia thymifolia & Thyme leaf & $\mathrm{Pd}$ & Whole plant & $\begin{array}{c}\text { Flies, mosquitoes } \\
\text { [57] }\end{array}$ \\
\hline Fioria vitifolia & $\begin{array}{c}\text { Grape leaved } \\
\text { mallow }\end{array}$ & $\mathrm{Ag}$ & Root, bark & Kills lice [58] \\
\hline Gloriosa superb & Flame lily & $\begin{array}{l}\mathrm{Ag}, \mathrm{Au}, \mathrm{Ce} \\
\mathrm{Cu}, \mathrm{Pt}, \mathrm{Pd}\end{array}$ & Leaf & Lice in the hair [59] \\
\hline Haldina cordifolia & Kadam & $\mathrm{Ag}$ & Bark & Insecticide [60] \\
\hline Hardwickia binate & Anjan & $\mathrm{Zn}$ & Wood & Insecticide [61] \\
\hline Harpullia arborea & Tulip wood & $\mathrm{Zn}, \mathrm{Sn}$ & Bark & Leech repellant [62] \\
\hline Holarrhena pubescens & Indrajao & $\mathrm{Ag}$ & Flower and seed & Insecticides [63] \\
\hline Hyptis suaveolens & American mint & $\mathrm{Ag}, \mathrm{Cu}$ & Twig & Repel bed bugs [64] \\
\hline Kalanchoe integra & Never die & $\mathrm{Au}, \mathrm{Cu}$ & Leaf & Insecticide [65] \\
\hline Lagenandra ovate & Malayan sword & $\mathrm{Ag}$ & Whole plant & Insecticide [66] \\
\hline Lavandula bipinnata & Lavender & $\mathrm{Zn}$ & Whole plant & Insect repellant [67] \\
\hline Lavendula lawii & Lavender & $\mathrm{Ag}$ & Whole plant & Insect repellant [68] \\
\hline Leonotis nepetifolia & Klip dagga & $\mathrm{Ag}$ & Leaf & $\begin{array}{c}\text { Housefly repellant } \\
\text { [69] }\end{array}$ \\
\hline Leucas aspera & Thumbai & $\mathrm{Ag}, \mathrm{Ce}, \mathrm{Cu}$ & Whole plant & Insecticide [70] \\
\hline Lippia javanica & Fever tea & $\mathrm{Ag}$ & Leaf & Insecticide [71] \\
\hline Madhuca longifolia & Ilippai & $\mathrm{Ag}, \mathrm{Cu}, \mathrm{Au}$ & Seed, seed oil and cake & $\begin{array}{l}\text { Worm killer, insect } \\
\text { repellant [72] }\end{array}$ \\
\hline Melaleuca leucadendron & Cajuput tree & $\mathrm{Au}, \mathrm{Ag}$ & Oil & $\begin{array}{c}\text { Mosquito repellant } \\
{[73]}\end{array}$ \\
\hline Melia azadarach & China berry & $\mathrm{Ag}$ & Fruit and seed & Insecticide [74] \\
\hline Melia volkensii & Melia & $\mathrm{Ag}$ & Fruit pulp & Termites [75] \\
\hline Millettia extensa & Benth & $\mathrm{Ag}$ & Root & Insecticide [76] \\
\hline Mimosa pudica & Shame plant & $\begin{array}{c}\mathrm{Ag}, \mathrm{Zn}, \mathrm{Fe}, \mathrm{Au}, \\
\mathrm{Cu}\end{array}$ & Leaf & $\begin{array}{c}\text { Veterinary } \\
\text { woundmaggots [77] }\end{array}$ \\
\hline Mundulea sericea & Cork bush & $\mathrm{Zn}$ & Seed,root and bark & insecticide [78] \\
\hline Nigella sativa & Black seed & $\mathrm{Ag}, \mathrm{Zn}$ & Seed & Pesticide [79] \\
\hline Ocimum americanum & Hoary basil & $\mathrm{Ag}$ & Whole plant & Insecticide [80] \\
\hline Ocimum gratissimum & Ram tulsi & $\mathrm{Ag}, \mathrm{Au}$ & Whole plant & Insect repellant [81] \\
\hline $\begin{array}{l}\text { Ocimum } \\
\text { kilimandscharicum }\end{array}$ & Camphor Basil & $\mathrm{Ag}$ & Leaves, flower & Mosquito, fleas [82] \\
\hline Ocimum tenuiflorum & Tulsi & $\mathrm{Ag}$ & Whole plant & Insect repellant [83] \\
\hline Peganum harmala & Wild rue & $\mathrm{Ag}, \mathrm{Zn}$ & Root & $\begin{array}{c}\text { Mosquito repellant } \\
\text { [84] }\end{array}$ \\
\hline Psidia punctulata & Mpepe, & $\mathrm{Ti}, \mathrm{Cu}$ & Leaves & $\begin{array}{c}\text { Lice, fleas, mites } \\
{[85]}\end{array}$ \\
\hline Pongamia pinnata & Indian beech & $\mathrm{Ag}$ & Seed, root, and seed oil & $\begin{array}{c}\text { Repellant and } \\
\text { insecticide [86] }\end{array}$ \\
\hline Riccinu scommunis & Castor bean & $\mathrm{Ag}, \mathrm{Au}$ & Seed oil & $\begin{array}{c}\text { Flies repellant, rice } \\
\text { moth and rice } \\
\text { weevil [87] }\end{array}$ \\
\hline Ruta graveolens & Herb of grace & $\mathrm{Zn}, \mathrm{Ag}$ & Whole plant & Insects [88] \\
\hline Sarcostmma viminale & Caustic vine & $\mathrm{Ag}$ & Leaf & White ants [89] \\
\hline
\end{tabular}




\begin{tabular}{|c|c|c|c|c|}
\hline Plant name & Common name & NPs Present & Part of the plant used & Application \\
\hline $\begin{array}{l}\text { Securidaca } \\
\text { longepedunculata }\end{array}$ & Violet tree & $\mathrm{Ag}$ & Whole plant & $\begin{array}{c}\text { Stored grain pest } \\
{[90]}\end{array}$ \\
\hline Senna didymobotrya & Popcorn senna & $\mathrm{Ag}$ & Leaves & Nematodes [91] \\
\hline Solanum incanum & Sodom apple & $\mathrm{Ag}$ & Fruit, leaf & Insecticides [92] \\
\hline Stephania japonica & Snake vine & $\mathrm{Au}, \mathrm{Ag}$ & Whole plant & $\begin{array}{l}\text { Strong poison to } \\
\text { frogs [93] }\end{array}$ \\
\hline Strychnos nux vomica & Poison nut & $\mathrm{Zn}, \mathrm{Au}, \mathrm{Ag}$ & Fruit and seed & White ants [94] \\
\hline Strychnos spinosa & Monkey orange & $\mathrm{Ag}$ & Whole plant & Insecticides [95] \\
\hline Symplytum officinale. & Common Comfrey & $\mathrm{Ag}$ & Leaf, root & Insecticides [96] \\
\hline Tagetes minuta & Wild marigold & $\mathrm{Ag}$ & Leaf, flower & Insecticides [97] \\
\hline $\begin{array}{l}\text { Tanacetum } \\
\text { cinerariifolium }\end{array}$ & Pyrethrum & $\mathrm{Ag}, \mathrm{Au}$ & Flower & $\begin{array}{c}\text { Bees and insects } \\
{[98]}\end{array}$ \\
\hline Teclea nobilis & Small Fruited teclea & $\mathrm{Ag}$ & Bark and leaves & Insecticides [99] \\
\hline Tephrosia purpurea & Fish poison & $\mathrm{Ag}, \mathrm{Au}$ & Wood, roots & $\begin{array}{l}\text { Cotton and woolen } \\
\text { cloth moths [100] }\end{array}$ \\
\hline Tithonia diversifolia & Marigold & $\mathrm{Ag}$ & Leaf, flower & Insecticides [101] \\
\hline Trachylobium ammi & Ajwain & $\mathrm{Ag}, \mathrm{Zn}$ & Seed & $\begin{array}{c}\text { Mosquito repellant } \\
\text { [102] }\end{array}$ \\
\hline $\begin{array}{l}\text { Trigonellasfoenum- } \\
\text { graecum }\end{array}$ & Fenugreek & $\mathrm{Au}, \mathrm{Ag}$ & Seed & $\begin{array}{c}\text { Insect repellant } \\
{[103]}\end{array}$ \\
\hline Vernonia amygdalina & Bitter leaf & $\mathrm{Ag}$ & Leaf & Insecticides [104] \\
\hline $\begin{array}{l}\text { Vernonia } \\
\text { anthelminticum }\end{array}$ & Kalijiri & $\mathrm{Zn}, \mathrm{Au}$ & Seed & Fleas [105] \\
\hline Vitex negundo & Chaste tree & $\mathrm{Ag}, \mathrm{Zn}$ & Leaf & $\begin{array}{c}\text { Insect repellant } \\
{[106]}\end{array}$ \\
\hline Vitex trifolia & Arabian lilac & $\mathrm{Ag}, \mathrm{Zn}, \mathrm{Au}$ & Leaf & $\begin{array}{c}\text { Insect repellant } \\
{[107]}\end{array}$ \\
\hline
\end{tabular}

\section{Conclusions}

This review paper provides an idea for future research to help the environment and humankind. Researchers have focused their attention on understanding the mechanism of synthesis of biopesticides and botanical-based nanoparticles for controlling pest, to prevent disease, and to protect our environment instead of chemical pesticides. Laboratory research, as well as field experiments, are encouraged to use nanoparticle-based insecticides to control pests and disease. Hence, the result shows that there are several ways to find an alternative source in the protection of human and environmental welfare.

\section{Funding}

This research received no external funding.

\section{Acknowledgments}

We acknowledge the support of the Department of Biotechnology, Adhiyamaan College of Engineering (Autonomous), Hosur, Tamil Nadu, India.

\section{Conflicts of Interest}

The authors declare no conflict of interest.

\section{References}

1. Misra, A.N.; Misral, M.; Singh, R. Nanotechnology in agriculture and food industry. Int J Pure Appl Sci Technol 2013, 16, 1-9.

2. Stadler, T.; Buteler, M.; Weaver, D.K. Novel use of nanostructured alumina as an insecticide. Pest management science 2010, 66, 577-579, https://doi.org/10.1002/ps.1915.

3. Owolade, O.F.; Ogunleti, D.O.; Adenekan, M.O. Titanium dioxide affects disease development and yield of edible cowpea. Agric Food Chem 2008,7, 2942-2947. 
4. Wen, L.X.; Ding, H.M.; Wang, J.X.; Chen, J.F. Porous hollow silica nanoparticles as carriers for controlled delivery of ibuprofen to small intestine. Journal of nanoscience and nanotechnology 2006, 6, 31393144,https://doi.org/10.1166/jnn.2006.410.

5. Devkumar. C.; Dureja. P.Global News on Pesticides.Pesticide Res. Journ 2002,14, 365-370.

6. Nag, S.K.; Raikwar, M.K. Persistent organochlorine pesticide residues in animal feed. Environmental monitoring and assessment2011,174, 327-335,https://doi.org/10.1007/s10661-010-1460-1.

7. Dureja, P.; Johnson, S. Photodegradation of azadirachtin-A: A neem-based pesticide. Current Science 2000,79, 1700-1703.

8. Ganesan, R.M.; Gurumallesh Prabu, H. Synthesis of gold nanoparticles using herbal Acorus calamus rhizome extract and coating on cotton fabric for antibacterial and UV blocking applications. Arabian Journal of Chemistry 2015, 12,2166-2174,https://doi.org/10.1016/j.arabjc.2014.12.017.

9. Ahmad, B.; Khan, I.; Shireen, F.; Bashir, S.; Azam, S.Green synthesis, characterisation and biological evaluation of AgNPs using Agave americana, Mentha spicata and Mangifera indica aqueous leaves extract. IET Nanobiotechnology 2016, 10, 281-287,https://doi.org/10.1049/iet-nbt.2015.0053.

10. Wardani, M.; Yulizar, Y.; Abdullah, I.; Bagus Apriandanu, D. Synthesis of NiO nanoparticles via green route using Ageratum conyzoides L. leaf extract and their catalytic activity. IOP Conference Series: Materials Science and Engineering 2019,509, https://doi.org/10.1088/1757-899X/509/1/012077.

11. Umar, H.; Kavaz, D.; Rizaner, N. Biosynthesis of zinc oxide nanoparticles using Albizia lebbeck stem bark, and evaluation of its antimicrobial, antioxidant, and cytotoxic activities on human breast cancer cell lines. International journal of nanomedicine 2018, 14, 87-100,https://doi.org/10.2147/IJN.S186888.

12. Jayakumarai, G.; Gokulpriya, C.; Sudhapriya, R.; Sharmila, G.; Muthukumaran, C. Phytofabrication and characterization of monodisperse copper oxide nanoparticles using Albizia lebbeck leaf extract. Applied Nanoscience 2015, 5, 1017-1021, https://doi.org/10.1007/s13204-015-0402-1.

13. Tippayawat, P.; Phromviyo, N.; Boueroy, P.; Chompoosor, A.Green synthesis of silver nanoparticles in aloe vera plant extract prepared by a hydrothermal method and their synergistic antibacterial activity. PeerJ 2016,4,e2589,https://doi.org/10.7717/peerj.2589.

14. Kasithevar, M.; Saravanan, M.; Prakash, P.; Kumar, H.; Ovais, M.; Barabadi, H.; Shinwari, Z.K. Green synthesis of silver nanoparticles using Alysicarpus monilifer leaf extract and its antibacterial activity against MRSA and CoNS isolates in HIV patients. Journal of Interdisciplinary Nanomedicine 2017, 2,131141,https://doi.org/10.1002/jin2.26.

15. Balavigneswaran, C.K.; Sujin Jeba Kumar, T.; Moses Packiaraj, R.; Prakash, S. Rapid detection of Cr(VI) by AgNPs probe produced by Anacardium occidentale fresh leaf extracts. Applied Nanoscience 2014, 4, 367-378,https://doi.org/10.1007/s13204-013-0203-3.

16. Begum,S.R.; Rao,D.M.; Reddy, P.D.S. Role of Green Route Synthesized Silver Nanoparticles in Medicinal Applications with Special Reference to Cancer Therapy. Biosci Biotech Res Asia2018,15,http://dx.doi.org/10.13005/bbra/2686.

17. Parthiban, E.; Manivannan, N.; Ramanibai, R.; Mathivanan, N. Green synthesis of silver-nanoparticles from Annona reticulata leaves aqueous extract and its mosquito larvicidal and anti-microbial activity on human pathogens. Biotechnology Reports2019,21, https://doi.org/10.1016/j.btre.2018.e00297.

18. Vivek, R.; Thangam, R.; Muthuchelian, K.; Gunasekaran, P.; Kaveri, K.; Kannan, S. Green biosynthesis of silver nanoparticles from Annona squamosa leaf extract and its in vitro cytotoxic effect on MCF-7 cells. Process Biochemistry2012, 47, 2405-2410,http://dx.doi.org/10.1016/j.procbio.2012.09.025.

19. Kumar, G.;Badoni, P.P.Arisaema tortuosum Leaf Extract Mediated Synthesis of Silver Nanoparticles, Characterization and their Antibacterial Activity. Asian J. Research Chem2018,11,419-422.

20. Doss,A. Biosynthesis and characterization of nanoparticles from three Aristolochia species. world journal of pharmacy and pharmaceutical sciences 2015, 4,1094-1104.

21. Yu, C.; Tang, J.; Liu, X.; Ren, X.; Zhen, M.; Wang, L. Green Biosynthesis of Silver Nanoparticles Using Eriobotrya japonica (Thunb.) Leaf Extract for Reductive Catalysis. Materials 2019,12,https://doi.org/10.3390/ma12010189.

22. Vijayakumar, M.; Priya, K.; Nancy, F.; Noorlidah, A.; Ahmed, A. Biosynthesis, characterisation and antibacterial effect of plant-mediated silver nanoparticles using Artemisia nilagirica. Industrial crops and products 2013, 41,235-240,https://doi.org/10.1016/j.indcrop.2012.04.017.

23. Ahmed, S.; Saifullah Ahmad, M.; Swami, B.; Ikram, S. Green synthesis of silver nanoparticles using Azadirachta indica aqueous leaf extract. Journal of Radiation Research and Applied Sciences 2016,9, 17,http://dx.doi.org/10.1016/j.jrras.2015.06.006.

24. Kataria, B.; Shyam, V.; Kaushik, B.; Vasoya, J.; Joseph, J.; Savaliya, C.; Kumar, S.; Parikh, S.; Thakar, C.; Pandya, D.; Ravalia, A.; Markna, J.; Shah, N. Green synthesis of silver nanoparticle using Bambusa arundinacea leaves. Proceedings of the International Conference on Functional Oxides and Nanomaterials2017, https://doi.org/10.1063/1.4982127.

25. Kyomuhimbo, H.D.; Michira, I.N.; Mwaura, F.B.; Derese, S.; Feleni, U.; Iwuoha, E.I. Silver-zinc oxide nanocomposite antiseptic from the extract of Bidens pilosa. SN Applied Sciences2019,1,https://doi.org/10.1007/s42452-019-0722-y. 
26. Benelli, G.;Govindarajan, M.; Rajeswary, M.; Senthilmurugan, S.; Vijayan, P.; Alharbi, N.S.; Kadaikunnan, S.; Khaled, J.M. Larvicidal activity of Blumea eriantha essential oil and its components against six mosquito species, including Zika virus vectors: the promising potential of (4E,6Z)-allo-ocimene, carvotanacetone and dodecyl acetate. Parasitology research 2017, 116, 1175-1188,https://doi.org/10.1007/s00436-017-5395-0.

27. Kora, A.J.; Sashidhar, R.; Arunachalam, J. Aqueous extract of gum olibanum (Boswellia serrata): A reductant and stabilizer for the biosynthesis of antibacterial silver nanoparticles. Process Biochemistry 2012,47, 1516-1520,https://doi.org/10.1016/j.procbio.2012.06.004.

28. Khan, S.; Ismail, M.; Khan, T.; Hussain, F.; Majid, A.; Iqbal, T.; Tasleem, F.; Wahab, Z.; Fayaz, M.; Rahim, T.; Khan, Y. Green synthesis of Brassica campestris mediated silver nanoparticles, their antibacterial and antioxidant activities. 2018, 6, 1943-1949.

29. Das, M.; Smita, S.S.Biosynthesis of silver nanoparticles using bark extracts of Butea monosperma (Lam.) Taub. and study of their antimicrobial activity. Appl Nanosci2018. 8,10591067,https://doi.org/10.1007/s13204-018-0721-0.

30. Gawade, V.V.; Gavade, N.L.; Shinde, H.M.; Babar, S.B.; Kadam, A.N.; Garadkar, K.M. Green synthesis of $\mathrm{ZnO}$ nanoparticles by using Calotropis procera leaves for the photodegradation of methyl orange. J Mater Sci: Mater Electron2017, 28,14033-14039, https://doi.org/10.1007/s10854-017-7254-2.

31. Singh, P.; Pandit, S.; Garnæs, J.; Tunjic, S.; Mokkapati, V.R.; Sultan, A.; Thygesen, A.; Mackevica, A.; Mateiu, R.V.; Daugaard, A.E.; Baun, A.; Mijakovic, I. Green synthesis of gold and silver nanoparticles from Cannabis sativa (industrial hemp) and their capacity for biofilm inhibition. International journal of nanomedicine 2018,13, 3571-3591,https://doi.org/10.2147/IJN.S157958.

32. Yuan, C.G.; Huo, C.; Yu, S.; Gui, B. Biosynthesis of gold nanoparticles using Capsicum annuum var. grossum pulp extract and its catalytic activity. Physica E: Low-dimensional Systems and Nanostructures2017,85, 19-26,https://doi.org/10.1016/j.physe.2016.08.010.

33. Nair, R.S., Snima, K.S.; Kamath, R.C.; Nair, S.V.; Lakshmanan, V.K.Synthesis and Characterization of Careya Arborea Nanoparticles for Assessing Its in Vitro Efficacy in Pancreatic Cancer Cells.Journal of Natural Products 2015, 8.

34. Rathnasamy, R.; Thangasamy, P.; Thangamuthu, R.; Sampath, S.; Alagan, V.Green synthesis of ZnO nanoparticles using Carica papaya leaf extracts for photocatalytic and photovoltaic applications. J Mater Sci: Mater Electron2017, 28,10374-10381,https://doi.org/10.1007/s10854-017-6807-8.

35. Joshi, N.; Jain, N.; Pathak, A.; Singh, J.; Prasad, R.; Upadhyaya, C.P. Biosynthesis of silver nanoparticles using Carissa carandas berries and its potential antibacterial activities. Journal of Sol-Gel Science and Technology2018, 86,682-689,https://doi.org/10.1007/s10971-018-4666-2.

36. Adesuji, E.T.; Oluwaniyi, O.O.; Adegoke, H.I.; Moodley, R.; Labulo, A.H.; Bodede, O.S.; Oseghale, C.O. Investigation of the Larvicidal Potential of Silver Nanoparticles against Culex quinque fasciatus: A Case of a Ubiquitous Weed as a Useful Bioresource. Journal of Nanomaterials2016,2016, 111,https://doi.org/10.1155/2016/4363751.

37. Nasrollahzadeh, M.; Issaabadi, Z.; Sajadi, S.M. Green synthesis of a $\mathrm{Cu} / \mathrm{MgO}$ nanocomposite by Cassytha filiformis L. extract and investigation of its catalytic activity in the reduction of methylene blue, congo red and nitro compounds in aqueous media. RSC Advances2018, 8, 37233735,https://doi.org/10.1039/C7RA13491F.

38. Haritha, E.; Roopan, S. M.; Madhavi, G.; Elango, G.; Al-Dhabi, N.A.; Arasu, M.V. Green chemical approach towards the synthesis of SnO2 NPs in argument with photocatalytic degradation of diazo dye and its kinetic studies. Journal of photochemistry and photobiology. B, Biology 2016, 162, 441447,https://doi.org/10.1016/j.jphotobiol.2016.07.010.

39. Huang, J.; Li, Q.; Sun, D.; Lu, Y.; Su, Y.; Yang, X.; Wang, H.; Wang, Y.; Shao, W.; He, N.; Hong, J.; Chen, C. Biosynthesis of silver and gold nanoparticles by novel sundried Cinnamomum camphora leaf. Nanotechnology2007, 18.

40. Sujitha, M.V.; Kannan, S. Green synthesis of gold nanoparticles using Citrus fruits (Citrus limon, Citrus reticulata and Citrus sinensis) aqueous extract and its characterization. Spectrochimica acta. Part A, Molecular and biomolecular spectroscopy2013, 102, 15-23,https://doi.org/10.1016/j.saa.2012.09.042.

41. Sarkar, D. Synthesis Of Plant-Mediated Silver Nanoparticles Using Commiphora Wightii (Guggul) Extract And Study Their Antibacterial Activities Against Few Selected Organisms. World Journal of Pharmacy and Pharmaceutical Sciences 2017, 1418-1425,https://doi.org/10.20959/wjpps20174-8924.

42. Ioset, J.R.; Marston, A.; Gupta, M.P.; Hostettmann, K. Antifungal and Larvicidal Compounds from the Root Bark of Cordia alliodora.Journal of Natural Products2000, 63,424-426, https://doi.org/10.1021/np990393j.

43. Abdel-Wahab, D.A.; Othman, N.A.R.M.; Hamada, A.M. Effects of copper oxide nanoparticles to Solanum nigrum and its potential for phytoremediation. Plant Cell Tiss Organ Cult2019, 137,525-539, https://doi.org/10.1007/s11240-019-01588-5.

44. Panda, S.K.; Dutta, S.K.; Bastia, A.K. Antibacterial activity of Croton roxburghii Balak. against the enteric pathogens. Journal of advanced pharmaceutical technology \& research 2010, 1, 419422,https://doi.org/10.4103/0110-5558.76442. 
45. Haryani, Y.; Nabella, I.; Yuharmen, Y.; Kartika, G.F. Synthesis of Silver Nanoparticles from Cucumis melo L. and Assessment of Its Antimicrobial Properties. Pharmacology and Clinical Pharmacy Research2018, 3, 46-50,https://doi.org/10.15416/pcpr.v3i2.18109.

46. Zhao, L.;Peralta-Videa, J.R.; Rico, C.M.; Hern andez-Viezcas, J.A.; Sun, Y.; Niu, G.; Servin, A.; Nunez, J.E.; Duarte-Gardea, M.; Gardea-Torresdey, J.L. $\mathrm{CeO}_{2}$ and $\mathrm{ZnO}$ nanoparticles change the nutritional qualities of cucumber (Cucumis sativus). Journal of agricultural and food chemistry2014, 62, 27522759,https://doi.org/10.1021/jf405476u.

47. Shameli, K.; Ahmad, M. B.; Zamanian, A.; Sangpour, P.; Shabanzadeh, P.; Abdollahi, Y.; Zargar, M. Green biosynthesis of silver nanoparticles using Curcuma longa tuber powder. International journal of nanomedicine2012, 7, 5603-5610,https://doi.org/10.2147/IJN.S36786.

48. Naghdi, S.; Sajjadi, M.; Nasrollahzadeh, M.; Rhee, K.Y.; Sajadi, S.M.; Jaleh, B. Cuscuta reflexa leaf extract mediated green synthesis of the $\mathrm{Cu}$ nanoparticles on graphene oxide/manganese dioxide nanocomposite and its catalytic activity toward reduction of nitroarenes and organic dyes. Journal of the Taiwan Institute of Chemical Engineers2018, 86, 158-173,https://doi.org/10.1016/j.jtice.2017.12.017.

49. Kamarudin, N.; Jusoh, R.; Setiabudi, H.; Jusoh, N.; Jaafar, N.; Sukor, N. Cymbopogon nardus Mediated Synthesis of Ag Nanoparticles for the Photocatalytic Degradation of 2,4-Dicholorophenoxyacetic Acid. Bulletin of Chemical Reaction Engineering \& Catalysis 2019,14, 173181,https://doi.org/10.9767/bcrec.14.1.3321.173-181.

50. Firdhouse, M.J.; Lalitha, P. Biosynthesis of silver nanoparticles using the extract of Alternanthera sessilisantiproliferative effect against prostate cancer cells. Cancer nanotechnology2013,4, 137143,https://doi.org/10.1007/s12645-013-0045-4.

51. Kumar, V. A.; Ammani, K.; Jobina, R.; Subhaswaraj, P.; Siddhardha, B. Photo-induced and phytomediated synthesis of silver nanoparticles using Derris trifoliata leaf extract and its larvicidal activity against Aedes aegypti. Journal of photochemistry and photobiologyB Biology2017, 171,18,https://doi.org/10.1016/j.jphotobiol.2017.04.022.

52. Ahmad, N.; Sharma, S.; Singh, V.N.; Shamsi, S.F.; Fatma, A.; Mehta, B.R. Biosynthesis of Silver Nanoparticles from Desmodium triflorum: A Novel Approach Towards Weed Utilization. Biotechnology research international 2011,2011,https://doi.org/10.4061/2011/454090.

53. Ashri, A.; Yusof, M.; Jamil, M.; Abdullah, A.; Yusoff, S.; Arip, M.; Lazim, A. Physicochemical characterization of starch extracted from malaysian wild yam(dioscorea hispida dennst.). Emirates Journal of Food and Agriculture2014, 26, 652-658,https://doi.org/10.9755/ejfa.v26i8.17098.

54. Ravindran, C.P.; M. Manokari.; Mahipal S.; Shekhawat. Biogenic production of zinc oxide nanoparticles from aqueous extracts of Duranta erecta L. World Scientific News2016, 28, 30-40.

55. Rajkuberan, C.; Prabukumar, S.; Sathishkumar, G.; Wilson, A.; Ravindran, K.; Sivaramakrishnan, S. Facile synthesis of silver nanoparticles using Euphorbia antiquorum L. latex extract and evaluation of their biomedical perspectives as anticancer agents. Journal of Saudi Chemical Society2017,21,911919,https://doi.org/10.1016/j.jscs.2016.01.002.

56. Annamalai, A.; Christina, V. L.; Sudha, D.; Kalpana, M.; Lakshmi, P.T. Green synthesis, characterization and antimicrobial activity of Au NPs using Euphorbia hirta L. leaf extract. Colloids and surfaces. B, Biointerfaces 2013, 108,60-65,https://doi.org/10.1016/j.colsurfb.2013.02.012.

57. Nasrollahzadeh, M.; Sajadi, S.M. Green synthesis of Pd nanoparticles mediated by Euphorbia thymifolia L. leaf extract: Catalytic activity for cyanation of aryl iodides under ligand-free conditions. Journal of colloid and interface science2016, 469,191-195,https://doi.org/10.1016/j.jcis.2016.02.024.

58. Ghosh, D.; Mondal, A.K. Biosynthesis of Silver Nano-particle from Four Medicinally Important Taxa in South West Bengal, India. Journal of Nanoscience and Nanotechnology 2015, 5, 305-312.

59. Ashokkumar, S.; Ravi, S.; Velmurugan, S. Green synthesis of silver nanoparticles from Gloriosa superba L. leaf extract and their catalytic activity. Spectrochimica acta. Part A, Molecular and biomolecular spectroscopy 2013,115, 388-392,https://doi.org/10.1016/j.saa.2013.06.050.

60. Khan, S.U.; Anjum, S.I.; Ansari, M.J.; Khan, M.H.; Kamal, S.; Rahman, K.; Shoaib, M.; Man, S.; Khan, A.J.; Khan, S.U.; Khan, D. Antimicrobial potentials of medicinal plant's extract and their derived silver nanoparticles: A focus on honey bee pathogen. Saudi Journal of Biological Sciences 2019, 26, 18151834,https://doi.org/10.1016/j.sjbs.2018.02.010.

61. Gunaselvi, G.; Kulasekaren, V.; Gopal, V. Anti bacterial and antifungal activity of various leaves extracts of Hardwickia binata Roxb. (Caesalpinaceae). International Journal of PharmTech Research 2010, 2, 21832187.

62. Mohan, S.C.; Hemalatha, K.; Manivel, A.; Kumar, M.S. Synthesis and Characterization of $\mathrm{Sn} / \mathrm{ZnO}$ Nanoparticles for Removal of Organic Dye and Heavy Metal. International Journal of Biological Chemistry2018, 12, 1-7,http://dx.doi.org/10.3923/ijbc.2018.1.7 .

63. VenkataSubbaiah,K.P.;Savithramma,N. Characterization and validation of silver nanoparticles from holarrhena pubescens - an important ethnomedicinal plant of kurnool district, andhra pradesh, india. World journal of pharmacy and pharmaceutical sciences 2013, 2, 6288-6300.

64. Elumalai, D.; Hemavathi, M.; Deepaa, C. V.; Kaleena, P.K. Evaluation of phytosynthesised silver nanoparticles from leaf extracts of Leucas aspera and Hyptis suaveolens and their larvicidal activity against 
malaria, dengue and filariasis vectors. Parasite epidemiology and control2017, 2, 1526,https://doi.org/10.1016/j.parepi.2017.09.001.

65. Patel, M.H.; Vashi, K.D.; Minocheherhomji, F.P.; Nagnae, R. Evaluation of antimicrobial activity and mutagenicity of copper nanoparticle synthesized using green chemistry. International journal of recent scientific research 2019, 10, 31196-31200.

66. Bokaeian, M.; Fakheri,B.A.; Mohasseli, T.; Saeidi, S. Antibacterial Activity of Silver Nanoparticles Produced by Plantago Ovata Seed Extract Against Antibiotic Resistant Staphylococcus aureus.Int J Infect2015, 2, e22854,https://dx.doi.org/10.17795/iji-22854.

67. Shaikh, R.; Pund, M.; Dawane, A.; Iliyas, S. Evaluation of Anticancer, Antioxidant, and Possible Antiinflammatory Properties of Selected Medicinal Plants Used in Indian Traditional Medication. Journal of traditional and complementary medicine2014, 4, 253-257,https://doi.org/10.4103/2225-4110.128904.

68. Kulkarni, R.R.; Pawar, P.V.; Joseph, M.P.; Akulwad, A.K.; Sen, A.; Joshi, S.P. Lavandula gibsoni and Plectranthus mollis essential oils: chemical analysis and insect control activities against Aedes aegypti, Anopheles sfttephensi and Culex quinquefasciatus.J Pest Sci2013,86,713718,https://doi.org/10.1007/s10340-013-0502-1.

69. Al-Sheddi, E.S.; Farshori, N.N.; Al-Oqail, M.M.; Al-Massarani, S.M.; Saquib, Q.; Wahab, R.; Musarrat, J.; Al-Khedhairy, A.A.; Siddiqui, M.A. Anticancer Potential of Green Synthesized Silver Nanoparticles Using Extract of Nepeta deflersiana against Human Cervical Cancer Cells (HeLA). Bioinorganic chemistry and applications 2018, 2018,https://doi.org/10.1155/2018/9390784.

70. Malleshappa, J.; Nagabhushana, H.; Sharma, S.; Vidya, Y.; Anantharaju, K.; Prashantha, S.; Prasad, B.D.; Naika, H.R.; Lingaraju, K.; Surendra, B. Leucas aspera mediated multifunctional CeO2 nanoparticles: Structural, photoluminescent, photocatalytic and antibacterial properties. Spectrochimica Acta Part A: Molecular and Biomolecular Spectroscopy2015, 149, 452-462,https://doi.org/10.1016/j.saa.2015.04.073.

71. Kumar, S.; Singh, M.; Halder, D.; Mitra, A. Lippia javanica: a cheap natural source for the synthesis of antibacterial silver nanocolloid. Appl Nanosci2016,6,1001-1007,https://doi.org/10.1007/s13204-015-05076.

72. Sharma, M.; Yadav, S.; Ganesh, N.; Srivastava, M.M.; Srivastava, S. Biofabrication and characterization of flavonoid-loaded Ag, Au, Au-Ag bimetallic nanoparticles using seed extract of the plant Madhuca longifolia for the enhancement in wound healing bio-efficacy. Prog Biomater2019, 8,5163,https://doi.org/10.1007/s40204-019-0110-0.

73. Souza, M.; Lopes, L.; Bonez, P.; Gündel, A.; Martinez, D.; Sagrillo, M.; Giongo, J.; Vaucher, R.; Raffin, R.; Boligon, A.; Santos, R. Melaleuca alternifolia nanoparticles against Candida species biofilms. Microbial Pathogenesis2017, 104,125-132,https://doi.org/10.1016/j.micpath.2017.01.023.

74. Anbu, P.; Murugan, K.; Madhiyazhagan, P.; Dinesh, D.; Subramaniam, J.; Panneerselvam, C.; Suresh, U.; Alarfaj, A.A.; Munusamy, M.A.; Higuchi, A.; Hwang, J. S.; Kumar, S.;Nicoletti, M.; Benelli, G. Greensynthesised nanoparticles from Melia azedarach seeds and the cyclopoid crustacean Cyclops vernalis: an eco-friendly route to control the malaria vector Anopheles stephensi?Natural product research2016, 30, 2077-2084,https://doi.org/10.1080/14786419.2015.1114935.

75. Kamau, R.W.; Juma, B.F.; Baraza, L.D. Antimicrobial compounds from root, stem bark and seeds of Melia volkensii.Natural product research 2016, 30, 1984-1987,https://doi.org/10.1080/14786419.2015.1101104.

76. Panda, S.K.; Mohanta, Y.K.; Padhi, L.; Park, Y.H.; Mohanta, T.K.; Bae, H. Large Scale Screening of Ethnomedicinal Plants for Identification of Potential Antibacterial Compounds. Molecules2016, 21, https://doi.org/10.3390/molecules21030293.

77. Fatimah, I.; Pradita, R.Y.; Nurfalinda, A. Plant Extract Mediated of ZnO Nanoparticles by Using Ethanol Extract of Mimosa Pudica Leaves and Coffee Powder. Procedia Engineering2016,148,4348,https://doi.org/10.1016/j.proeng.2016.06.483.

78. Chaithong, U.; Choochote, W.; Kamsuk, K.; Jitpakdi, A.; Tippawangkosol, P.; Chaiyasit, D.; Champakaew, D.; Tuetun, B.; Pitasawat, B. Larvicidal effect of pepper plants on Aedes aegypti (L.) (Diptera: Culicidae). Journal of vector ecology : journal of the Society for Vector Ecology2006, 31, 138-144, https://doi.org/10.3376/1081-1710(2006)31[138:leoppo]2.0.co;2.

79. Amooaghaie, R.; Saeri, M.R.; Azizi, M. Synthesis, characterization and biocompatibility of silver nanoparticles synthesized from Nigella sativa leaf extract in comparison with chemical silver nanoparticles. Ecotoxicology and environmental safety2015, 120, 400-408,https://doi.org/10.1016/j.ecoenv.2015.06.025.

80. Anuradha , G.; Syama , B.; Ramana, M.V. Ocimum americanum L. leaf extract mediated synthesis of silver nano particles: A noval approach towards weed utilisation. Archives of Applied Science Research 2014, 6, 59-64.

81. Das, B.; Dash, S.K.; Mandal, D.; Ghosh, T.; Chattopadhyay, S.; Tripathy, S.; Das, S.; Dey, S.K.; Das, D.; Roy, S. Green synthesized silver nanoparticles destroy multidrug resistant bacteria via reactive oxygen species mediated membrane damage. Arabian Journal of Chemistry2017, 10, 862876,https://doi.org/10.1016/j.arabjc.2015.08.008.

82. Selvarani,S.; Vinayaga, P.M.; Ramalakshmi,M.; Padmapriya, S.; Abirami, M. Ocimum Kilimandscharicum Leaf Extract Engineered Silver Nanoparticles and Its Bioactivity. J Nanomater Mol Nanotechnol2016, 5,https://doi.org/10.4172/2324-8777.1000179.

https://nanobioletters.com/ 
83. Singhal, G.; Bhavesh, R.; Kasariya, K.; Sharma, A.R.; Singh, R.P. Biosynthesis of silver nanoparticles using Ocimum sanctum (Tulsi) leaf extract and screening its antimicrobial activity. J Nanopart Res 2011, 13, 29812988,https://doi.org/10.1007/s11051-010-0193-y.

84. Fazlzadeh, M.; Khosravi, R.; Zarei, A. Green synthesis of zinc oxide nanoparticles using Peganum harmala seed extract, and loaded on Peganum harmala seed powdered activated carbon as new adsorbent for removal of $\mathrm{Cr}(\mathrm{VI})$ from aqueous solution. Ecological Engineering 2017,103,180190,https://doi.org/10.1016/j.ecoleng.2017.02.052.

85. Zinjarde, S.S.; Bhargava, S.Y.; Kumar, A.R. Potent $\alpha$-amylase inhibitory activity of Indian Ayurvedic medicinal plants. BMC Complement Altern Med2011,11, https://doi.org/10.1186/1472-6882-11-5.

86. Paul, M.; Londhe, V.Y. Pongamia pinnata seed extract-mediated green synthesis of silver nanoparticles: Preparation, formulation and evaluation of bactericidal and wound healing potential. Applied Organometallic Chemistry2018, 33,https://doi.org/10.1002/aoc.4624.

87. Ojha, S.; Sett, A.; Bora, U. Green synthesis of silver nanoparticles by Ricinus communis var. carmencita leaf extract and its antibacterial study. Advances in Natural Sciences: Nanoscience and Nanotechnology 2017,8, https://doi.org/10.1088/2043-6254/aa724b.

88. Lingaraju, K.; Raja Naika, H.; Manjunath, K.; Basavaraj, R.B.; Nagabhushana, H.; Nagaraju, G.; Suresh, D. Biogenic synthesis of zinc oxide nanoparticles using Ruta graveolens (L.) and their antibacterial and antioxidant activities. Appl Nanosci 2016, 6, 703-710,https://doi.org/10.1007/s13204-015-0487-6.

89. Kannan B, N.; Thoppil, J. Plant-mediated synthesis of silver nanoparticles by two species of Cynanchum L. (Apocynaceae): A comparative approach on its physical characteristics. Int. J. Nano Dimens 2018, 9,104111.

90. Ojewole, J.A.O. Analgesic, anti-inflammatory and hypoglycaemic effects of Securidaca longepedunculata (Fresen.)[Polygalaceae] root-bark aqueous extract. Inflammopharmacol2008,16,174-181, https://doi.org/10.1007/s10787-007-0016-7.

91. Vijayakumari, A.; Sinthiya, A. Biosynthesis of Phytochemicals Coated Silver Nanoparticles Using Aqueous Extract of Leaves of Cassia alata - Characterization, Antibacterial and Antioxidant Activities. International Journal of Pharmaceutical and Clinical Research2018, 10, 138-149.

92. Venkat Kumar, S.; Karpagambigai, S.; Jacquline Rosy, P.; Rajeshkumar,S. Phyto-Assisted Synthesis of Silver Nanoparticles using Solanum Nigrum and Antibacterial Activity Against Salmonella Typhi and Staphylococcus Aureus. Mechanics, Materials Science Engineering Journal2017, 9.

93. Kar, P.K.; Murmu, S.; Saha, S.; Tandon, V.; Acharya, K. Anthelmintic Efficacy of Gold Nanoparticles Derived from a Phytopathogenic Fungus, Nigrospora oryzae. PLoS ONE2014, 9,https://doi.org/10.1371/journal.pone.0084693.

94. Steffy, K.; Shanthi, G.; Maroky, A.S.; Selvakumar, S. Synthesis and characterization of ZnO phytonanocomposite using Strychnos nux-vomica L. (Loganiaceae) and antimicrobial activity against multidrug-resistant bacterial strains from diabetic foot ulcer. Journal of Advanced Research 2018, 9, 6977,https://dx.doi.org/10.1016\%2Fj.jare.2017.11.001.

95. Isa, A.I.; Awouafack, M.D.; Dzoyem, J.P.; Aliyu, M.; Magaji, R.A.; Ayo, J.O.; Eloff, J.N. Some Strychnos spinosa (Loganiaceae) leaf extracts and fractions have good antimicrobial activities and low cytotoxicities. BMC Complement Altern Med2014, 14,456, https://doi.org/10.1186/1472-6882-14-456.

96. Singh, H.; Du, J.; Singh, P.; Yi, T.H. Role of green silver nanoparticles synthesized from Symphytum officinale leaf extract in protection against UVB-induced photoaging. J Nanostruct Chem 2018, 8, 359368,https://doi.org/10.1007/s40097-018-0281-6.

97. Shahzadi, I.; Shah, M.M. Acylated flavonol glycosides from Tagetes minuta with antibacterial activity. Frontiers in Pharmacology2015, 6,https://doi.org/10.3389/fphar.2015.00195.

98. Kitherian, S. Nano and Bio-nanoparticles for Insect Control. Research Journal of Nanoscience and Nanotechnology2016, 7,1-9,http://dx.doi.org/10.3923/rjnn.2017.1.9.

99. Mahamadi, C.; Wunganayi, T. Green synthesis of silver nanoparticles using Zanthoxylum chalybeum and their antiprolytic and antibiotic properties. Cogent Chemistry 2018, 4,https://doi.org/10.1080/23312009.2018.1538547.

100. Srikar, S.K.; Giri, D.D.; Pal, D.B.; Mishra, P.K.; Upadhyay, S.N. Green Synthesis of Silver Nanoparticles: A Review. Green and Sustainable Chemistry 2016, 6, 34-56,http://dx.doi.org/10.4236/gsc.2016.61004.

101. Tran, T.T.T.; Vu, T.T.H.; Nguyen, T.H. Biosynthesis of silver nanoparticles using Tithonia diversifolia leaf extract and their antimicrobial activity. Materials Letters 2013,105,220223,https://doi.org/10.1016/j.matlet.2013.04.021.

102. Chouhan, N.; Ameta, R.; Meena, R.K. Biogenic silver nanoparticles from Trachyspermum ammi ( Ajwain ) seeds extract for catalytic reduction of $\mathrm{p}$-nitrophenol to $\mathrm{p}$-aminophenol in excess of $\mathrm{NaBH} 4$. Journal of Molecular Liquids 2017, 230, 74-84,https://doi.org/10.1016/j.molliq.2017.01.003.

103. Aswathy Aromal, S.; Philip, D. Green synthesis of gold nanoparticles using Trigonella foenum-graecum and its size-dependent catalytic activity. Spectrochimica acta. Part A, Molecular and biomolecular spectroscopy2012, 97, 1-5,https://doi.org/10.1016/j.saa.2012.05.083. 
104. Widyaningtyas, A.L.; Yulizar, Y.; Apriandanu, D.O.B. Ag2O nanoparticles fabrication by Vernonia amygdalina Del. leaf extract: synthesis, characterization, and its photocatalytic activities. IOP Conference Series: Materials Science and Engineering 2019, 509,https://doi.org/10.1088/1757-899x/509/1/012022.

105. Karthikeyan, A.; Siva, G.; Sujitha, S.; Rex, D.; Chidambaranathan, N. Antihyperglycemic activity of the ethanolic seed extract of Vernonia anthelminticum willd. International Journal of Green Pharmacy2008, 2 , 209.

106. Prabhu, D.; Arulvasu, C.; Babu, G.; Manikandan, R.; Srinivasan, P. Biologically synthesized green silver nanoparticles from leaf extract of Vitex negundo L. induce growth-inhibitory effect on human colon cancer cell line HCT15.Process Biochemistry2013, 48, 317-324,https://doi.org/10.1016/j.procbio.2012.12.013.

107. Elumalai, K.; Velmurugan, S.; Ravi, S.; Kathiravan, V.; Raj, G.A. Bio-approach: Plant mediated synthesis of $\mathrm{ZnO}$ nanoparticles and their catalytic reduction of methylene blue and antimicrobial activity. Advanced Powder Technology2015, 26, 1639-1651,https://doi.org/10.1016/j.apt.2015.09.008. 